\title{
Intermediarios y poder de mercado en los mercados agrícolas de México: un enfoque de teoría de juegos
}

\section{Rubén Chavarín Rodríguez*}

\section{RESUMEN}

El objetivo del trabajo consiste en modelar los efectos del poder de mercado presente en los eslabones de intermediación en los mercados agrícolas de México; para cumplirlo, se elaboró un conjunto de modelos de teoría de juegos. La conclusión general establece que cuando un intermediario agrícola tiene poder de mercado en la fijación de su precio de venta, no sólo genera efectos en los eslabones siguientes de la cadena del producto sino que repercute negativamente en los productores; tal resultado se mantiene aun cuando éstos cuentan con poder negociador en la venta, a pesar de que el intermediario se comporte como precioaceptante al comprar; cuando, además, hay poder de mercado en la fijación del precio de compra, este resultado se refuerza. Sin embargo, el establecimiento de contratos anticipados provoca que el intermediario sacrifique una parte de sus beneficios para incentivar la participación del productor.

Palabras clave: poder de mercado, mercados agrícolas, teoría de juegos, países emergentes.

Clasificación JEL: L11, L14, L79, Q12.

Profesor Investigador en el Departamento de Estudios Regionales, CUCEA-Universidad de Guadalajara, Jalisco, México. Correos electrónicos: ruben.chavarin@cucea.udg.mx, rachavarin@yahoo.com. 


\section{ABSTRACT}

\section{Intermediaries and market power in the agricultural markets of Mexico: a game theory approach}

The aim of this work is to model the effects of intermediaries' market power established in agricultural markets in Mexico. To accomplish this purpose, a set of game theory models have been built. The general conclusion indicates that when an agricultural intermediary has market power by setting his ask price, not only produces effects on next linkages in the product chain but also generates negative repercussions on the producers. This result holds even when the producer has bargaining power selling his product, and despite the intermediary behaves as a price-taker when he buys the product. This result is reinforced when intermediary also has market power by setting bid prices. However, when establishing anticipated contracts, the intermediary has to sacrifice a share of his benefits to incentivize the participation of the producer in the market.

Keywords: market power, agricultural markets, game theory, emging markets

JEL CLassification: L11, L14, L79, Q12

\section{INTRODUCCIÓN}

La intermediación en una economía resulta necesaria para que diversos bienes, luego de ser producidos, lleguen más fácilmente a las manos de quienes desean consumirlos (Belleflamme y Peitz, 2010: 613); de no haber intermediarios sería muy difícil que, por ejemplo, una zanahoria producida en el estado de Puebla fuera comprada de manera rutinaria por un consumidor de Ciudad Juárez, Chihuahua, luego de haber recorrido más de $1900 \mathrm{~km}$ por carretera ${ }^{1}$.

Un intermediario agrícola, como agente económico, realiza operaciones de compra-venta de granos, cereales, frutas, verduras y legumbres frescos; la esencia de su negocio reside en obtener beneficios económicos a partir de los márgenes derivados de la compra-venta de los productos, una vez descontados todos sus costos. En el proceso de

Según los reportes del Servicio de Información Agroalimentaria y Pesquera, las zanahorias consumidas en Ciudad Juárez proceden del estado de Puebla (SIAP, 2015). 
intermediación se añade valor al producto por el transporte, la distribución mayorista y el acopio; éste puede incluir almacenamiento, clasificación, lavado, empacado y etiquetado. En casos especializados, el intermediario resulta capaz de formar una red de transporte y almacenamiento en frío; en otros, provee de insumos y financiamiento al productor.

A diferencia de otros mercados, en el ámbito agrícola existen miles de productores para cada artículo; una parte muy significativa comercializa directamente a un intermediario. Por ejemplo, en el caso de la papa de origen mexicano, $55 \%$ de los productores declara venderle a un intermediario, $24 \%$ a un mayorista y casi $3 \%$ a una cadena comercial; de acuerdo con la definición amplia utilizada en el presente trabajo, tales compradores son tipos distintos de intermediarios por lo que, en realidad, aproximadamente $82 \%$ de los productores de papa vende su producto a un intermediario. En el caso de la cebada, sucede lo mismo con cerca del 90\% del total nacional de productores (Cofece, 2016: 52).

Gran parte de los productores agrícolas de México venden sus productos a un intermediario por la especialización de este tipo de agentes económicos, pero otra parte de los productores se ven en la necesidad de vender a un intermediario porque carecen de una infraestructura básica de almacenamiento y transporte, o bien, porque no cuentan con ciertos conocimientos acerca de los procesos de comercialización; de acuerdo con datos del Censo Agrícola, Ganadero y Forestal 2007 del Instituto Nacional de Estadística, Geografía e Informática (INEGI), 31\% de los productores nacionales de calabacita consideraba que la comercialización resultaba el principal problema para desarrollar su actividad; lo mismo sucedía para $27 \%$ de los productores de papa, el $24 \%$ de los de jitomate y el $25 \%$ de los de naranja (Cofece, 2016: 38). Esta información destaca en tanto muestra en cierta medida el lado negativo del fenómeno de la intermediación, ya que hay evidencia diversa respecto a que muchos intermediarios aportan poco valor agregado y ejercen prácticas abusivas de compra en perjuicio de los productores; en tal sentido, De la Rosa (2016) refiere el caso de los agricultores de manzanilla del Estado de México, que en 2016 recibían un pago promedio de 12 pesos por kilogramo de flor de manzanilla deshidratada, cuando el consumidor final pagaba, dependiendo de la marca, entre 516 y 2,750 pesos por la misma cantidad, sólo que en presentación de bolsas de té de un gramo; así, el margen de comercialización entre el 
productor y el minorista fue de 4,200\% o más. En esta época en que la producción y comercialización de alimentos se ha vuelto tan relevante, y en la que México se ha convertido en un exportador importante de productos agrícolas hacia Estados Unidos, resulta paradójico que los agricultores sean los menos beneficiados (Calleja-Pinedo, 2007: 17).

De acuerdo con la Comisión Federal de Competencia Económica (Cofece), los problemas de asimetría en el poder de negociación entre productores e intermediarios pueden agravarse en la medida en que estos últimos sean de mayor tamaño, o bien, cuando los productores se encuentren en las siguientes situaciones: $i$ ) tengan una escala de producción pequeña, ii) no estén organizados de manera adecuada con otros productores, iii) estén menos informados que sus compradores sobre los precios y otras variables del mercado, y iv) tengan acceso a un número limitado de comercializadores (Cofece, 2016: 281). A su vez, tales conflictos conducen directamente a temas abordados por la organización industrial, como el poder de mercado: la capacidad de un agente económico de fijar precios por encima de los costos marginales; comúnmente, se estudia en la parte de las ventas, aunque también puede darse por el lado de las compras. Este último caso constituye el punto de interés del presente trabajo: explorar el funcionamiento de los mercados agrícolas a partir de la relación de los productores con los intermediarios, donde es posible que estos últimos ejerzan poder de mercado al adquirir productos del campo.

Ello es relevante por varias razones. En primer lugar, los productos agrícolas representan la base de la alimentación en México y el resto del mundo; en particular, en nuestro país la dieta tradicional de la población se ha integrado principalmente por maíz, frijol, chile, arroz y diversas frutas, verduras y legumbres, entre otros. Debe considerarse, entonces, que el control discrecional de los precios de los productos en los eslabones de intermediación puede perjudicar a los productores agrícolas, a quienes usan esos artículos como insumos y, por supuesto, a quienes los consumen frescos o procesados; de hecho, en el periodo 2005-2016, el crecimiento acumulado de los precios de los alimentos en México (66.8\%) ha sido bastante mayor que en el promedio de los países de la Organización para la Cooperación y el Desarrollo Económicos (28.6\%), y también superior al de las mercancías no alimenticias en el propio país: 40.3\% (OECD, 2016). Tal como señala la Cofece (2016: 103), este incremento en México puede deberse a ciertas influencias interna- 
cionales y a efectos de la depreciación del tipo de cambio mexicano; a su vez, la mayor inflación en los alimentos en territorio nacional sugiere la existencia de factores propios del sector agroalimentario, como la conducta de los intermediarios agrícolas.

En segundo lugar, en México el gasto en alimentos representa un porcentaje muy significativo de los ingresos totales, como ocurre con las familias que se encuentran en el decil de más bajos ingresos: el gasto en alimentos representó $79 \%$ en $2014^{2}$. Así, destaca que cualquier incremento en los precios de los productos agrícolas debido a situaciones contrarias a la competencia y libre concurrencia tiene repercusiones socioeconómicas muy sensibles.

En tercer lugar, falta conocimiento preciso acerca de los eslabones de intermediación de los productos agrícolas; como señala la Cofece (2016: 73, 281), se cuenta con datos sobre la cantidad de productores, pero no respecto a la de intermediarios: sólo se sabe que su número es notablemente menor, por lo que habría un mayor grado de concentración en estos eslabones, sobre todo en determinados nichos. Tampoco hay información sistematizada en cuanto a los volúmenes manejados por los distintos intermediarios y muy poca en torno a los precios, incluyendo la manera como se determinan; en consecuencia, actualmente no es posible calcular, en la mayor parte de los casos, los márgenes de comercialización obtenidos por los intermediarios. La suma de los vacíos referidos provoca una comprensión insuficiente sobre las conductas económicas en estos eslabones del mercado agrícola nacional.

Por las razones señaladas, se pretende contribuir al estudio de los efectos generados por la presencia o ausencia del poder de mercado de los intermediarios en las conductas de los agentes económicos interrelacionados con ellos. Para ello, se parte de la exposición de un conjunto de modelos de teoría de juegos, regidos por un enfoque de organización industrial que facilita el análisis de las conductas económicas de los individuos y empresas que interactúan en los mercados y, además, permite ofrecer elementos para la discusión de aspectos regulatorios.

En el apartado 1 se brindan algunos fundamentos conceptuales sobre la intermediación y se mencionan algunos enfoques teóricos rele-

2 Este dato procede de la Encuesta Nacional de Ingresos y Gastos de los Hogares 2014 del INEGI (Cofece, 2016: 91). 
vantes para su estudio; en especial, se da una breve explicación del enfoque de organización industrial seguido en el presente trabajo. En el apartado 2 se presentan los modelos de teoría de juegos, divididos en dos partes: a) casos en los que no hay contratos anteriores a las transacciones entre productores e intermediarios, y b) casos en donde sí existen contratos anticipados. Por último, se incluyen las conclusiones. Al final del documento se ofrece un apéndice con ejemplos del cálculo de los pagos de los modelos de teoría de juegos estudiados en el apartado 2.

\section{Algunos elementos teóricos SObre LA INTERMEDIACIÓN}

Los intermediarios surgen ante la dificultad de que los productores y consumidores de bienes comercien directamente entre sí. La sola oportunidad de hacer transacciones de compra-venta mediante un intermediario genera valor para ciertos compradores y vendedores, en comparación a tener que hacerlo por intercambio directo en un mercado totalmente descentralizado (Belleflamme y Peitz, 2010: 614-616); por ello, la función de la intermediación en una economía se reconoce necesaria. De hecho, en ciertas circunstancias, puede tener diversas ventajas sobre el intercambio directo: a) reduce costos de transacción, b) diversifica riesgos, c) reduce costos de búsqueda, d) alivia la selección adversa, y e) mitiga el riesgo moral y el oportunismo (Spulber, 1999: xiii).

Existen dos mecanismos básicos de operación para un intermediario: a) compra-venta de bienes, con la obtención de un margen de comercialización; y b) operación de una plataforma de compra-venta, con el cobro de un cargo por el uso de la plataforma; ambos modelos se presentan en la Figura 1. El presente trabajo se enfoca en el modelo de intermediación mediante compra-venta (parte A de la Figura 1) ${ }^{3}$, mecanismo empleado en el sector agrícola; implica dos precios de referencia fundamentales para el intermediario: el de compra (bid price), al cual adquiere el bien del productor en el eslabón anterior, y el de venta (ask price), al cual suministra el mismo producto en el eslabón siguiente; la diferencia entre ellos constituye el margen de intermediación o de

\footnotetext{
Hay otro tipo de intermediarios, llamados brokers o comisionistas, quienes actúan en representación de terceros a cambio de una comisión, pero este tipo de intermediarios pueden entenderse como parte del modelo asentado en el inciso a).
} 
comercialización. Entonces, la esencia de este negocio consiste en obtener beneficios económicos a partir de los márgenes de intermediación de los productos, una vez descontados todos sus costos.

Figura 1

LOS DOS MODELOS DE INTERMEDIACIÓN

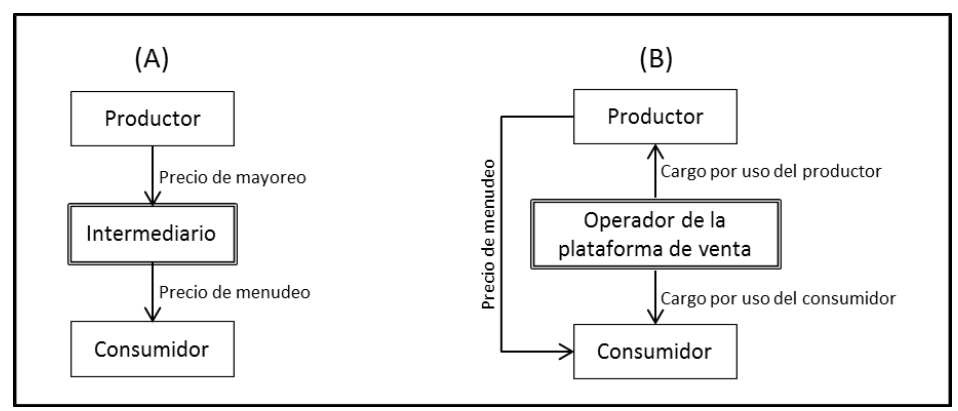

Fuente: Belleflamme y Peitz (2010: 617).

Actualmente hay varios enfoques teóricos de desarrollo reciente sobre la intermediación, como los modelos en redes (intermediation networks), que estudian los efectos del fenómeno mediante estructuras de red, donde los resultados económicos obtenidos por los individuos se encuentran determinados por su ubicación y relaciones, así como por su estructura (Condorelli y Galeotti, 2016; Manea, 2016). Otro enfoque importante es el de la microestructura de mercado, también conocido como teoría de la intermediación de la empresa, desarrollado a partir de la idea de que los mercados alcanzan el equilibrio a través de la determinación estratégica de los precios por parte de los intermediarios; de acuerdo con esto, cualquier tipo de empresa opera como tal y elige sus precios de venta (no los toma como dados) para obtener ingresos y racionar la demanda del consumidor; a su vez, selecciona sus precios de compra para estimular a sus proveedores y mantener bajos los costos de los inventarios (Spulber, 1996; 1999). Aunque esta teoría considera, en sentido amplio, a cualquier empresa como intermediaria, cuando se aplica a los mercados de mayoreo y menudeo se vuelve una teoría especializada de la intermediación, en el sentido estricto del término.

En el enfoque de organización industrial, base de este trabajo, las empresas se desenvuelven en entornos de competencia imperfecta y desarrollan estrategias para desempeñarse con el mayor éxito posible. Con frecuencia, en la búsqueda de mayores beneficios, las empresas 
desarrollan poder de mercado, que es la capacidad de fijar precios distintos a los que se obtendrían en un ámbito donde prevalecieran la competencia y la libre concurrencia; cuando ello sucede, puede decirse que es precio-decisora: entonces enfrenta una curva de demanda con pendiente negativa, diferente a la curva de demanda horizontal que encara una empresa tomadora de precios, lo cual implica que ésta maximiza sus beneficios con base en el patrón mostrado en las ecuaciones 1 y $2^{4}$.

$$
\begin{aligned}
& \pi(q)=p(q) q-c(q) \\
& d \pi(q) / d q=p(q)+q^{\prime}(q)-c^{\prime}(q)=0
\end{aligned}
$$

Donde: $\mathrm{p}(\mathrm{q})=$ función inversa de demanda de la empresa, $\mathrm{q}=$ cantidad vendida de producto, $\mathrm{c}(\mathrm{q})$ = función de costos de la empresa.

La ecuación 2 muestra que cuando la empresa con poder de mercado incrementa su producción hay dos efectos: $p(q)$ significa que sus ingresos se incrementan porque la empresa recibe el precio por la unidad adicional vendida; y $q p^{\prime}(q)$ implica que, al producir una unidad extra, la empresa se mueve hacia abajo en el eje de los precios, siguiendo su curva de demanda, y entonces debe cobrar un precio menor a todas las unidades vendidas. En particular, esto hace que las empresas con poder de mercado limiten la cantidad que venden a un nivel inferior al que lo harían si fueran tomadoras de precios; la combinación de comercializar menor cantidad y cobrar mayor precio genera un costo de oportunidad a la sociedad, un resultado muy conocido.

Los efectos señalados se magnifican cuando la empresa también ejerce poder de mercado como compradora; en un entorno de competencia y libre concurrencia, todos los costos de oportunidad derivados del poder de mercado serían ganancias de bienestar que irían hacia los consumidores.

Aquí se sigue un enfoque de organización industrial porque estudia la conducta de los intermediarios agrícolas ante la presencia o ausencia de poder de mercado, a partir de funciones de beneficios microeconómicas y modelación de teoría de juegos como herramientas de análisis;

4 Este tipo de ecuaciones aparecen en cualquier texto de organización industrial, v. gr. el texto de Tirole (1988: 66-67), aunque presenta una notación matemática distinta. 
al mismo tiempo, permite abordar los efectos de la interrelación entre productores agrícolas e intermediarios.

En los modelos mostrados en los apartados siguientes se asumen productos homogéneos, lo que refleja la situación de diversos productos agrícolas en la que aún no es tan relevante la diferenciación de características, como sucede con los comercializados en los mercados municipales y callejeros, sitios que representan un canal trascendente del consumo final en México. Sin embargo, se reconoce la creciente importancia de dicha diferenciación en otros segmentos de mercado, sobre todo en canales de distribución dirigidos hacia consumidores de estratos de ingresos altos, quienes demandan productos con ciertos atributos de calidad, que involucran aspectos de apariencia y conveniencia, así como de origen, que implican procesos productivos amigables con el medio ambiente y una mercantilización bajo ciertas condiciones como el comercio justo- (Sexton, 2013). Los modelos que incorporan tales distinciones en los productos incluyen un mayor número de estrategias y enfrentan la complicación de generar equilibrios múltiples difíciles de caracterizar en trabajos empíricos (Bonanno, Russo y Menapace, 2018); el caso de productos diferenciados no se contempla en el presente trabajo.

Finalmente, en la búsqueda de literatura realizada durante la presente investigación, no se encontró algún documento que trate la intermediación agrícola en México de la forma como aquí se realiza. Por supuesto, diversos estudios abordan con distintas técnicas el poder de mercado y sus efectos, pero con énfasis estrictamente teórico o aplicado a otros tipos de problemas económicos.

\section{ANÁLISIS DE TEORÍA DE JUEGOS DE LOS INTERMEDIARIOS AGRÍCOLAS}

\subsection{Los componentes de los modelos}

En algunos manuales básicos de economía (como Pindyck y Rubinfeld, 2009), los mercados agrícolas se ponen como ejemplo de una situación parecida a los de competencia perfecta, puesto que, se afirma, sus precios generalmente están determinados por el mercado; esta es una aseveración, que los autores plantean para fines didácticos, razonablemente válida para determinados productos, en ciertos lugares, en 
algún elemento de su cadena de comercialización. Sin embargo, estos mercados son más complejos de lo que aparentan debido a la presencia de uno o más eslabones de intermediación; en la cadena que siguen los productos agrícolas puede haber asimetrías de tamaño, recursos, capacidades y, en suma, de poder de negociación entre los diferentes agentes participantes. En términos económicos, las asimetrías pueden convertirse en poder de mercado, de modo que las empresas adquieran la capacidad de fijar precios (de compra o venta) distintos de los de un mercado competitivo.

Como se verá a continuación, la presencia o ausencia de poder de mercado es el elemento clave de los modelos elaborados que, además de permitir explicar este aspecto, conllevan otras ventajas. En particular, propician un análisis sencillo de los diferentes escenarios de negociación entre productores e intermediarios, así como de sus consecuencias económicas; adicionalmente, la forma en que se modela la presencia de contratos entre productores e intermediarios (sección 2.2.2) muestra que éstos contrarrestan, en cierta medida, las ventajas en poder de negociación de los intermediarios, quienes tienen la necesidad de asegurar el flujo de los productos en condiciones preestablecidas. Trabajos recientes sobre contratos y coordinación vertical señalan que los estudios basados en el enfoque de poder de mercado no reflejan este tipo de situaciones (Saxon, 2013), pero en el presente trabajo se observan resultados donde los efectos señalados de los contratos están presentes en un contexto de poder de mercado.

\subsubsection{Jugadores y estrategias de los modelos}

El análisis aquí incluido se ha enfocado en modelar la relación entre un productor (denominado $\mathrm{P}$ en los diagramas siguientes) y un intermediario (denominado I). El productor es cualquier individuo o empresa que siembra, cosecha y vende productos agrícolas; el intermediario, cualquier agente que realiza actividades de compra-venta de productos agrícolas, lo que incluye a un individuo, una empresa, un comercializador mayorista o una cadena comercial. Las posibles estrategias de estos jugadores son las siguientes:

Para el productor: (No, Si) que significan (actuar sin poder negociador, actuar con poder negociador). 
Para el intermediario: $(A, D)$ que significan (actuar como precioaceptante, actuar como precio-decisor).

Hay un tercer jugador en los modelos, que es la naturaleza (denominado $\mathrm{N}$ en los diagramas siguientes):

Para la naturaleza: $(B, R, M)$ que significan (enviar clima bueno durante el ciclo agrícola, enviar clima regular durante el ciclo agrícola, enviar clima malo durante el ciclo agrícola).

En primer lugar, que la naturaleza juegue clima bueno (B) significa que las condiciones ambientales fueron idóneas y se obtuvo una producción mayor a la promedio, de forma que hay más oferta del producto que la común y eso hace que bajen los precios. En segundo lugar, que la naturaleza juegue clima regular (R) implica que las condiciones ambientales estuvieron dentro del promedio y, por lo tanto, la producción y los precios también se ubican en la media. En tercer lugar, si la naturaleza juega clima malo (M), las condiciones ambientales fueron desfavorables en cierta medida (como cuando hay problemas de heladas, sequía o exceso de lluvias) y se obtuvo una producción inferior a la normal, de modo que hay menos oferta del producto que la común y eso hace que suban los precios. Cabe aclarar que en este estado del juego se considera una afectación moderada respecto al clima (R); no se toman en cuenta situaciones extraordinariamente malas porque, en ese caso, todos los jugadores resultarían perjudicados.

Como se sabe, la naturaleza juega sus estrategias con cierta probabilidad. En los modelos ilustrados en las figuras 2, 4, 6, y 8 se propone que la naturaleza juegue con probabilidad de $1 / 2$ el clima regular y con probabilidad de 1/4 cada uno de los otros escenarios (bueno y malo). Estos valores se han asignado para ilustrar que es más probable esperar un clima regular (es decir, promedio) y menos, climas fuera de la media. Sin embargo, también se resolvieron los modelos asignando probabilidades iguales (o sea, 1/3 a cada uno) a los tres tipos de clima; en todos los casos se llegó a los mismos equilibrios que en los modelos base (resultados no reportados). También se resolvieron los modelos asignando sucesivamente la probabilidad mayor $(1 / 2)$ al clima bueno y al malo; en casi todos los casos se llegó a los mismos equilibrios que en el modelo base (resultados no reportados); sólo se encontró la excepción referida en el apartado 2.2.2.1. Debido a que en casi todos los casos se consiguen los mismos equilibrios, independientemente del clima al que 
se asigne mayor probabilidad, las conclusiones no se alterarían a pesar de que las probabilidades de ocurrencia de los climas fueran aleatorias en lugar de determinísticas ${ }^{5}$.

\subsubsection{Los pagos de los modelos}

Los pagos de los modelos se refieren a los valores que adquieren las funciones de beneficios del productor e intermediario bajo cada combinación de estrategias; son resultado, en cada caso, de la maximización de dicha función. En el caso del intermediario, su función de beneficios puede asumir que es precio-aceptante o precio-decisor tanto en precio de compra (bid price) como de venta (ask price).

\subsubsection{Los pagos del intermediario}

El intermediario cuenta con cuatro tipos de funciones de beneficios, definidos por la posesión o carencia de poder de mercado al determinar sus precios de compra y venta; cabe aclarar que el tipo de funciones presentadas a continuación para los casos con poder de mercado muestran aquellas situaciones donde se tendría el máximo posible; hay, por supuesto, otras donde las empresas podrían competir de acuerdo con los modelos de Cournot o Stackelberg y presentar menor poder de mercado; para los propósitos del presente estudio, introducir ese tipo de funciones no cambiaría la elección de los equilibrios y sí generaría complicaciones innecesarias. Los cuatro tipos de funciones de beneficios del intermediario son los siguientes:

a) El intermediario es precio-aceptante en ambos eslabones:

$$
\pi^{I}=p q-w x-f
$$

b) El intermediario es precio-aceptante al comprar al productor y es precio-decisor al vender en el eslabón siguiente:

$$
\pi^{I}=p(q) q-w x-f
$$

5 Sin embargo, el tema de la forma de asignación de las probabilidades se volvería especialmente relevante si introdujéramos algún tipo de información asimétrica a los modelos (quizás en cuanto a la calidad), lo que conduciría a la necesidad de usar la regla de Bayes y obtener equilibrios bayesianos perfectos. 
c) El intermediario es precio-decisor al comprar al productor y es precio-aceptante al vender en el eslabón siguiente:

$$
\pi^{l}=p q-w(x) x-f
$$

d) El intermediario es precio-decisor en ambos eslabones:

$$
\pi^{I}=p(q) q-w(x) x-f
$$

Donde: $\mathrm{p}=$ precio al que vende el producto, $\mathrm{q}=$ cantidad vendida de producto, $\mathrm{w}=$ precio al que compra el producto al productor, $\mathrm{x}=$ cantidad comprada de producto al productor, $\mathrm{f}=$ costos fijos.

Las funciones de beneficios pueden reescribirse mediante el uso de una función de producción. En este caso, se usó la siguiente ${ }^{6}$ :

$$
q=f(x)=x
$$

Se propuso esta función de producción por tres razones: i) para reflejar la actividad de intermediación, ya que la función indica que el intermediario vende la misma cantidad de producto que compra; ii) para mostrar que hay rendimientos constantes a escala en la actividad del intermediario; y iii) para facilitar los cálculos derivados de la maximización de las funciones de beneficios. De esta manera, las funciones de beneficios del intermediario se reformulan como sigue:

a) El intermediario es precio-aceptante en ambos eslabones:

$$
\pi^{I}=p x-w x-f
$$

b) El intermediario es precio-aceptante al comprar al productor y es precio-decisor al vender en el eslabón siguiente:

$$
\pi^{I}=p(x) x-w x-f
$$

${ }_{6}^{6} \quad$ Esta función de producción es localmente cóncava, de modo que cumple con las propiedades exigidas para la maximización de beneficios: $\frac{d f(x)}{d x}>0, \frac{d^{2} f(x)}{d x^{2}} \leq 0$ 
c) El intermediario es precio-decisor al comprar al productor y es precio-aceptante al vender en el eslabón siguiente:

$$
\pi^{I}=p x-w(x) x-f
$$

d) El intermediario es precio-decisor en ambos eslabones:

$$
\pi^{I}=p(x) x-w(x) x-f
$$

Para encontrar soluciones explícitas resultantes de la maximización de las funciones y así facilitar el cálculo y presentación de los equilibrios de los modelos, se propuso una función inversa de demanda lineal:

$$
p(q)=1-q
$$

Esta función de demanda también puede reformularse a partir de la función de producción. Adicionalmente, se incluyó una función inversa de oferta del factor de producción, la cual indica la cantidad media por pagar para obtener un nivel dado de dicho factor; permite modelar las variaciones de la oferta del factor a partir de las elecciones hechas por la naturaleza: se desplaza hacia la derecha cuando hay condiciones climáticas buenas, lo que resulta en una mayor cantidad producida y una reducción de los precios; se desplaza hacia la izquierda cuando hay condiciones climáticas desfavorables, lo que genera una reducción en la cantidad producida y un aumento en los precios. Se plantea una función distinta para cada estado de la naturaleza:

a) Cuando la naturaleza juega clima bueno $(B)$ :

$$
w(x)=x
$$

b) Cuando la naturaleza juega clima regular $(R)$ :

$$
w(x)=x+1 / 4
$$

c) Cuando la naturaleza juega clima malo $(M)$ :

$$
w(x)=x+1 / 2
$$


Las funciones de beneficios incluyen costos fijos para reflejar que el intermediario debe incurrir en gastos relacionados con la integración de cierto tipo de infraestructura, ya sea de transporte, acopio, distribución mayorista o, incluso, para el manejo en frío de los productos. Una vez que se obtienen los valores de equilibrio para los precios y cantidades en cada función, se resuelve la forma reducida restando al final los costos fijos. En todos los casos se tomó un valor dado para $f$ de forma tal que reflejara el hecho de que un intermediario con poder de mercado, en uno o ambos eslabones, debería obtener beneficios mayores o iguales que cero. El valor usado en todos los casos fue de $f=1 / 40$.

Finalmente, en los casos en que el intermediario es precio-aceptante en el eslabón siguiente, debe hacer un cargo extra a su precio de venta para recuperar sus costos fijos ya que, de otra manera, tendría beneficios negativos iguales $a-f$ y perdería el estímulo económico para participar en el mercado. Con el cargo extra puede obtener beneficios iguales a cero, como sucede en los mercados competitivos.

\subsubsection{Los pagos del productor}

El productor cuenta con dos tipos de funciones de beneficios, definidos por la posesión o carencia de poder de negociación en la determinación de los precios a los que vende al intermediario. Cuando el productor no tiene poder negociador es precio-aceptante y toma los valores de $\mathrm{w}^{*} \mathrm{y} \mathrm{x}^{*}$ determinados en la solución óptima del intermediario en la misma rama del juego, que corresponden, respectivamente, al $\hat{\mathrm{p}}$ y $\hat{\mathrm{q}}$ del productor (se usa la designación $\hat{\mathrm{p}}, \hat{\mathrm{q}}$ para resaltar que se trata del productor, en contraste con los valores de equilibrio del intermediario que se designan como de $\left.\mathrm{p}^{*}, \mathrm{q}^{*}, \mathrm{w}^{*}, \mathrm{x}^{*}\right)$. Este primer caso se representa de la siguiente forma:

a) El productor no tiene poder de negociación:

$$
\pi^{P}=\bar{p} \hat{q}-\bar{c} \hat{q}
$$

En el segundo caso, el productor sí posee poder negociador en la determinación de los precios a los que vende al intermediario; en otras palabras, el productor pertenece a una organización, de manera que se beneficia de una negociación conjunta y puede contar con mayores recursos para acceder a más opciones de compra; así, no es sujeto de 
conductas monopsónicas. Aquí, en el cálculo de los beneficios óptimos del productor, se tomó un valor dado para $\overline{\mathrm{p}}$ ligeramente superior al precio correspondiente del mismo modelo cuando el productor es precio-aceptante, de forma tal que $\overline{\mathrm{p}}>\hat{\mathrm{p}} ;{ }^{7} \overline{\mathrm{p}}$ alude al valor dado $\overline{\mathrm{w}}$ que el intermediario toma cuando es precio-aceptante en la misma rama del juego. Con $\overline{\mathrm{w}}$ el intermediario obtiene el valor óptimo de $\mathrm{x}^{*}$, que a su vez representa q para el productor. Este segundo tipo de función de beneficios se representa de la siguiente forma:

b) El productor sí tiene poder de negociación:

$$
\pi^{P}=\hat{p} \hat{q}-\bar{c} \hat{q}
$$

Cuando en una misma rama del juego coincide que el productor posee poder negociador y el intermediario es precio-decisor, se considera que estos efectos se contrarrestan y que los jugadores negocian un precio igual al caso en que ambos son precio-aceptantes. También se probó una situación en la que, dado que ambos jugadores poseen poder negociador, mejor deciden no comerciar entre sí y cada uno obtiene beneficios iguales a cero; esto no se presenta en los modelos mostrados a continuación porque conduce a los mismos equilibrios que cuando ambos se consideran precio-aceptantes.

Para obtener una solución explícita y facilitar el cálculo de los equilibrios de los modelos, en los dos tipos de funciones de beneficios del productor se tomó un valor dado para c; se determinó a partir de un caso de referencia equivalente al de la competencia perfecta, donde productor e intermediario son precio-aceptantes como comprador y vendedor, respectivamente. En ese caso, el valor de $\bar{c}=1 / 2$ es consistente con el resultado de beneficios iguales a cero; se incluyó en todos los modelos del productor.

\subsection{Los tipos de modelos}

Los modelos se dividen en dos grupos principales: $i$ ) sin contratos anticipados entre productor e intermediario, y ii) con contratos anticipados entre productor e intermediario. En los dos grupos de modelos se cons-

Por ejemplo, en una situación en donde el productor era precio-aceptante y obtenía un $\hat{\mathrm{p}}=1 / 2$

$=0.50$, se propuso un $\overline{\mathrm{p}}=5 / 8=0.53$. 
truye una versión en donde el intermediario sí tiene poder de mercado en el eslabón siguiente, y otra donde carece de él.

Un aspecto importante vinculado con los modelos desarrollados a continuación es que, como ya se ha explicado, están construidos con base en un conjunto de condiciones que facilita su análisis y solución, como una curva de demanda lineal, rendimientos constantes a escala en los intermediarios y curvas de oferta de factores lineales, además de un par de supuestos acerca de ciertos valores dados de precios y costos. No obstante, este conjunto de condiciones es menos restrictivo de lo que puede parecer porque, a pesar de que se planteen cambios en la posición de las curvas y en los valores que se toman como dados en ciertas ramas de los juegos, los resultados principales no se modifican y permiten explicar de manera consistente distintas conductas de los agentes analizados.

\subsubsection{Modelos sin contratos anticipados entre productor e intermediario}

En estos modelos la naturaleza juega antes de que el productor y el intermediario acuerden los precios con los que habrán de comerciar; como las elecciones de la naturaleza suceden durante el periodo que va desde la siembra hasta la cosecha, tanto el productor como el intermediario son capaces de observarla y saber si finalmente eligió clima bueno (B), regular (R) o malo (M). Cuando el productor cosecha y está listo para vender, ya ha tomado las acciones para determinar si tendrá o no poder de negociación; entonces entra el intermediario, quien observa la elección de la naturaleza y del productor. Por lo tanto, estos juegos se pueden modelar de manera sucesiva, tal como se muestra en las figuras 2 y 3.

\subsubsection{El intermediario no tiene poder de mercado en el eslabón siguiente}

En este primer modelo, cuando toca el turno del intermediario, juega sin ejercer poder de mercado en el eslabón siguiente, es decir, maximiza sus beneficios de acuerdo con las ecuaciones 8 y 10, según sea el caso; la forma completa de este juego puede verse en el diagrama 2. En el apéndice que aparece al final del documento se muestra un ejemplo del 
cálculo de los beneficios del intermediario y el productor en una rama determinada de este juego.

Figura 2

JUEGO SIN CONTRATO ANTICIPADO DONDE EL INTERMEDIARIO NO TIENE PODER DE MERCADO EN EL ESLABÓN SIGUIENTE

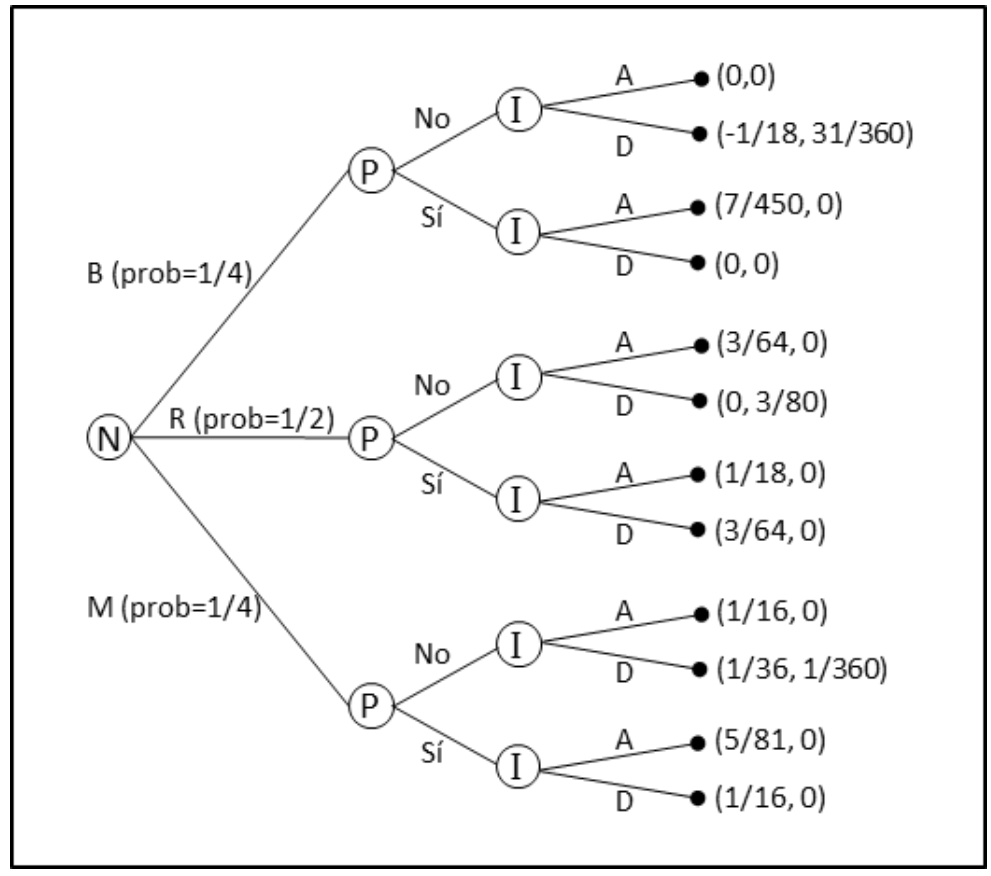

Fuente: Elaboración propia con base en las ecuaciones de los apartados 2.1.2.1 y 2.1.2.2.

\subsection{Equilibrios del modelo}

Aunque la Figura 2 muestra que la naturaleza dispone el clima $(R)$ con probabilidad igual a $1 / 2$, mientras que elige $(B)$ y $(M)$ con probabilidad de 1/4 cada uno, sólo sirve para tener un panorama completo del juego en sentido amplio, en tanto ambos jugadores la observan. En cambio, para efectos prácticos de encontrar los equilibrios del juego, éste se puede dividir en tres juegos independientes: los participantes juegan la parte superior cuando saben que hubo buen clima; la parte central, con regular; y la parte inferior, con malo (véase Figura 3). Como el juego se modeló de manera sucesiva, es necesario hallar uno o más equilibrios perfectos en subjuegos (EPS). 


\section{Clima bueno (B)}

En la parte superior del juego, el intermediario claramente elige $D$ sobre $A$; en cambio, en la parte baja el intermediario es indiferente entre elegir $A$ o $D$. Entonces, podría proponerse que el productor considere una distribución de probabilidad ( $p, 1-p)$ para elegir entre No y $S i$, pero no tiene caso porque $7 / 450>-1 /(18)$, y $0>-1 /(18)$; así, el productor siempre elegirá Sí; por lo tanto, hay dos EPS: $\{S i, A\},\{S i, D\}$.

\section{Figura 3}

JUEGOS DONDE EL INTERMEDIARIO NO TIENE PODER DE MERCADO EN EL ESLABÓN SIGUIENTE Y AMBOS JUGADORES OBSERVAN LA ELECCIÓN DE LA NATURALEZA

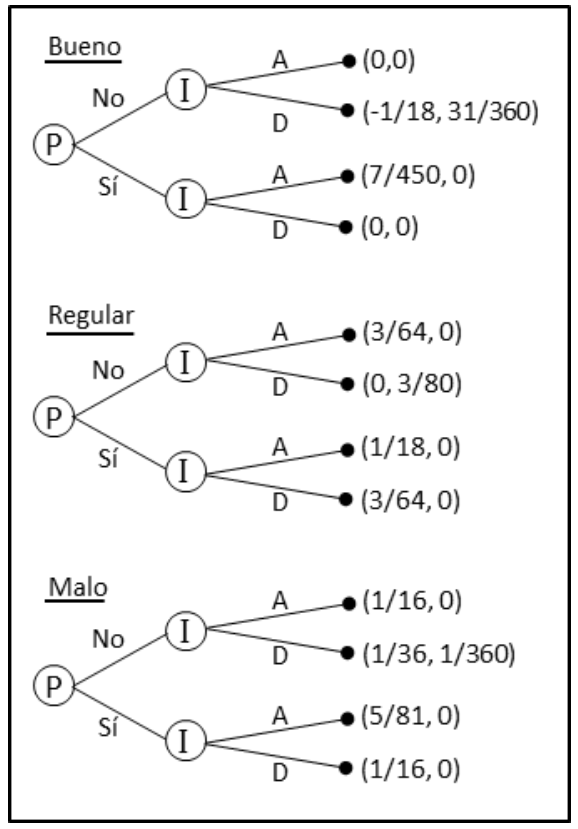

Fuente: Elaboración propia con base en las ecuaciones de los apartados 2.1.2.1 y 2.1.2.2.

\section{Clima regular $(R)$}

En la parte superior de este juego, el intermediario claramente elige $D$ sobre $A$; en cambio, en la baja, es indiferente entre elegir $A$ o $D$. Como $1 / 18>0$, y 3/64>0, entonces el productor siempre elegirá $S i$; por lo tanto, hay dos EPS: $\{S i, A\},\{S i, D\}$. 


\section{Clima malo (M)}

Al igual que en los casos anteriores, en la parte superior del juego el intermediario claramente elige $D$ sobre $A$; en cambio, en la baja, resulta indiferente entre elegir $A$ o $D$. Como 5/81 >1/36, y $1 / 16>1 / 36$, entonces el productor siempre elegirá $S i$; por lo tanto, hay dos EPS: $\{S i$, $A\},\{S i, D\}$.

Comparando con los otros estados de la naturaleza, con clima $(M)$ el productor logra su mejor situación en términos de beneficios; en el equilibrio $\{S i, A\}$ consigue $5 / 81$ y en $\{S i, D\}$ obtiene $1 / 16$. Como en ninguno de éstos el intermediario puede ejercer poder de mercado como comprador ni como vendedor, entonces en ambos termina con beneficios nulos. Se aclaró que en $(M)$ el productor alcanza su mejor situación en términos de beneficios (en comparación con los otros estados del clima) porque se considera una afectación moderada respecto al clima $(R)$; este efecto no se sostendría para situaciones del clima extraordinariamente malas que afectaran proporciones muy grandes de la producción. Este comentario vale para todos los modelos del documento.

Los resultados de los tres juegos analizados muestran que cuando no hay contratos anticipados y el intermediario carece de poder de mercado en el eslabón siguiente, un resultado de equilibrio es que el productor elige tener poder de negociación, lo que, como ya se señaló, implica actuar dentro de una organización o asociación.

\subsubsection{El intermediario sí tiene poder de mercado en el eslabón siguiente}

La diferencia fundamental con los modelos de la sección 2.2.1.1 es que, cuando toca el turno del intermediario, lo hace mediante el ejercicio del poder de mercado en el eslabón siguiente, es decir, maximiza sus beneficios de acuerdo con las ecuaciones 9 y 11, según sea el caso; la forma completa de este juego puede verse en la Figura 4. En el apéndice al final del documento se muestra un ejemplo del cálculo de los beneficios del intermediario y el productor en una rama determinada de este juego.

\subsection{Equilibrios del modelo}

La Figura 4 muestra que la naturaleza elige clima $(R)$ con probabilidad igual a $1 / 2$, mientras que elige $(B)$ y $(M)$ con probabilidad de $1 / 4$ cada uno; como ambos jugadores la observan, el diagrama sirve para tener 
un panorama completo del juego en sentido amplio. Al igual que en la sección 2.2.1.1, para efectos prácticos de encontrar los equilibrios del juego, éste se puede dividir en tres juegos independientes: los participantes juegan la parte superior cuando saben que hubo buen clima; la parte central, con clima regular; y la parte inferior, con clima malo (véase Figura 5). Los equilibrios que se buscan son EPS debido a que se trata de juegos que se desarrollan de manera sucesiva.

Figura 4

JUEGO SIN CONTRATO ANTICIPADO DONDE EL INTERMEDIARIO SÍ TIENE PODER DE MERCADO EN EL ESLABÓN SIGUIENTE

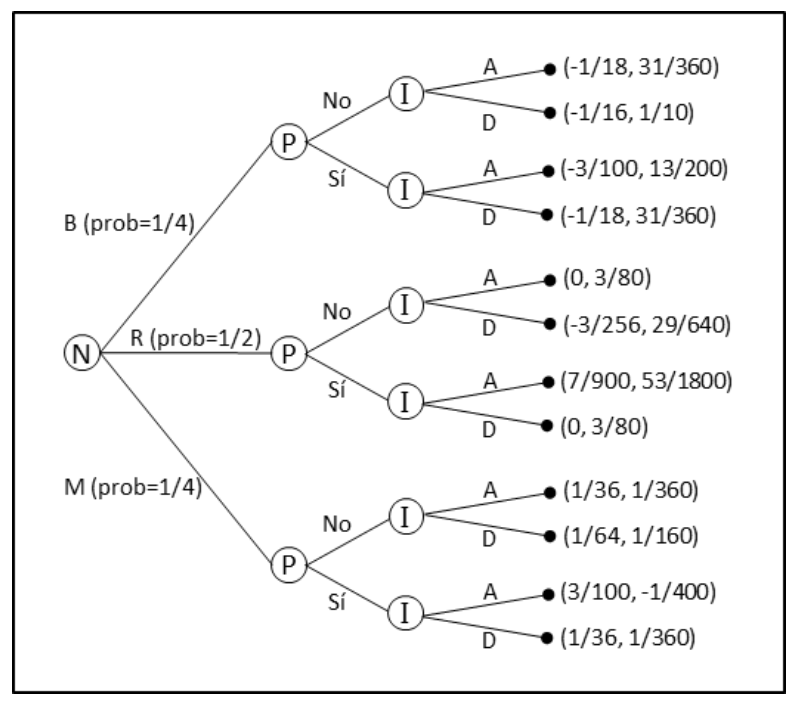

Fuente: Elaboración propia con base en las ecuaciones de los apartados 2.1.2.1 y 2.1.2.2.

\section{Clima bueno (B)}

Para el intermediario, $D$ resulta una estrategia estrictamente dominante y, por esta razón, elige $D$ sobre $A$ tanto en la parte superior como en la inferior del juego; en su turno, el productor elige $S i$, conduciendo al EPS: $\{S i, D\}$. Cabe recordar que en esta combinación de estrategias los poderes de negociación del productor y del intermediario se contrarrestan y negocian los precios de manera equivalente (o al menos cercana) a un caso en que ambos actúen como precio-aceptantes, aunque también el intermediario se ve favorecido en términos de beneficios debido a que ejerce poder de mercado en el eslabón siguiente. Además, 
el intermediario logra ganancias superiores con tal estado de la naturaleza: el clima bueno le permite comprar al productor a un precio inferior que bajo otras condiciones climáticas.

Debe notarse que, como D es una estrategia estrictamente dominante para el intermediario, cualquier desviación del productor de su estrategia de equilibrio conducirá a la combinación $\{$ No, D $\}$, situación en la que el intermediario ejerce el poder de mercado en ambos eslabones. Si las condiciones socioculturales, geográficas o económicas impiden que un productor pertenezca a una asociación de productores debidamente organizada para comercializar mejor los productos de los miembros, entonces el juego se irá a la rama donde el intermediario obtiene sus mayores beneficios $(1 / 10)$ y el productor, los peores $(-1 / 16)$.

Figura 5

JUEGOS DONDE EL INTERMEDIARIO SÍ TIENE PODER DE MERCADO EN EL ESLABÓN SIGUIENTE Y AMBOS JUGADORES OBSERVAN LA ELECCIÓN DE LA NATURALEZA

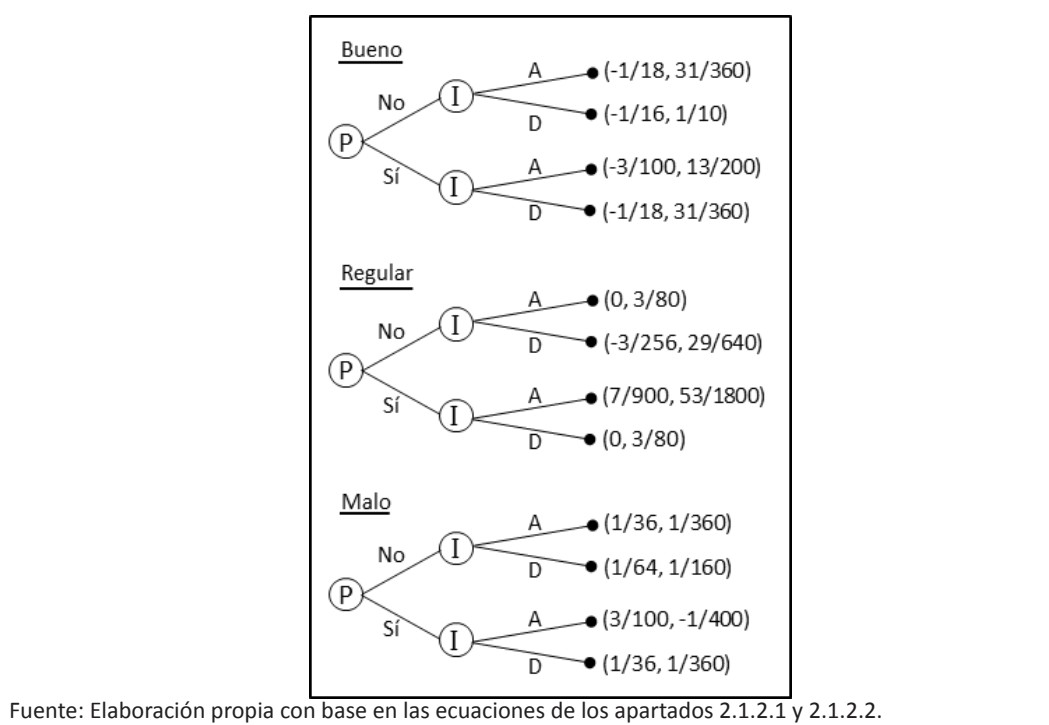

Clima regular $(R)$

Al igual que con clima $(B)$, en este caso $D$ implica una estrategia estrictamente dominante para el intermediario, de manera que elige $D$ sobre $A$ tanto en la parte superior como en la inferior del juego. En su turno, el productor elige $S i$ y conduce al EPS: $\{$ Sí, D $\}$. Como se llega al mismo equilibrio que con clima $(B)$, también sucede aquí que los poderes de 
negociación del productor e intermediario se contrarrestan y negocian los precios de manera equivalente o cercana a un caso en que ambos actúen como precio-aceptantes, aunque el intermediario ejerce poder de mercado en el eslabón siguiente. En comparación con los otros estados de la naturaleza, en el clima $(R)$ tanto el intermediario como el productor obtienen beneficios intermedios.

Como en el estado $(B)$, cualquier desviación del productor de su estrategia de equilibrio conducirá a la combinación $\{N o, D\}$, situación en la que el intermediario ejerce el poder de mercado en ambos eslabones, en beneficio propio y en perjuicio del productor.

\section{Clima malo (M)}

Tal como sucede con $(B)$ y $(R)$, en este caso $D$ constituye una estrategia estrictamente dominante para el intermediario: lo conduce a elegir $D$ sobre $A$ tanto en la parte superior como en la inferior del juego. En su turno, el productor elige $S i$ y conduce al EPS: $\{S i, D\}$, donde se contrarrestan los poderes de negociación del productor y el intermediario. Si se compara con los otros estados de la naturaleza, en $(M)$ el productor logra su mejor situación en términos de beneficios (en el equilibrio llega a obtener 1/36), mientras que para el intermediario la situación resulta menos favorable (en el equilibrio consigue 1/360) debido a que con clima adverso debe pagar precios proporcionalmente más altos al productor.

En coincidencia con los estados $(B)$ y $(R)$, cualquier desviación del productor de su estrategia de equilibrio conducirá a la combinación $\{N o, D\}$.

\subsubsection{Modelos con contratos anticipados entre productor e intermediario}

Estos modelos se refieren a los casos denominados "agricultura por contrato", ${ }^{8}$ donde el productor y el intermediario acuerdan los precios antes de que intervenga la naturaleza; su principal beneficio consiste en eliminar la incertidumbre respecto al precio para las transacciones,

\footnotetext{
Según la Organización de las Naciones Unidas para la Alimentación y la Agricultura (FAO), la agricultura por contrato es la operación por la que el productor vende al comprador antes de cosechar su producto, a través de la celebración de contratos de compra-venta, bajo condiciones específicas de precio, volumen, calidad, tiempo, lugar de entrega y condiciones de pago (Eaton y Shepherd, 2002).
} 
en tanto los contratos reflejan los montos y condiciones que se obtendrían ante escenarios climáticos promedio, es decir, de acuerdo con el estado de la naturaleza $(R)$. Por esta razón, en la construcción de los modelos se resuelve una función de beneficios especial del contrato, en donde el intermediario $\left(\pi^{I C}\right)$ o el productor $\left(\pi^{P C}\right)$, según corresponda, consideran como dado el valor de $\mathrm{w}^{*}$ equivalente al caso donde los participantes han observado que la naturaleza jugó $(R)$. En la función que maximiza el intermediario $\left(\pi^{I C}\right)$, éste toma el valor señalado de $w^{*}$ como uno dado $\bar{w}$, en la función que maximiza el productor $\left(\pi^{P C}\right)$, éste toma $w^{*}$ como un valor dado $\bar{p}$. En concreto, los pagos de los juegos representan la ganancia o pérdida resultante de la comparación entre la existencia o ausencia de contrato; por ello, al valor de los beneficios de la función especial del acuerdo se restan los que se habrían obtenido si no hubiera habido contrato, siempre a partir del estado de la naturaleza: a) para el intermediario: $\pi^{I C}-\pi^{I B}, \pi^{I C}-\pi^{I R}, \pi^{I C}-\pi^{I M}$; b) para el productor: $\pi^{P C}-\pi^{P B}, \pi^{P C}-\pi^{P R}, \pi^{P C}-\pi^{P M} .9$ Estos son los pagos de los juegos mostrados en las figuras 6 y 8 que, como puede observarse y ya que se modelan de manera sucesiva, consideran que el intermediario observa la elección del productor antes de negociar el precio del producto mediante un contrato y después interviene la naturaleza, por lo que los equilibrios son EPS.

\subsubsection{El intermediario no tiene poder de mercado en el eslabón siguiente}

En este primer caso de modelo de agricultura por contrato, cuando toca el turno de que participe el intermediario, lo hace sin ejercer poder de mercado en el eslabón siguiente: maximiza sus beneficios de acuerdo con las ecuaciones 8 y 10, según sea el caso; ello se presenta en la Figura 6.

\subsection{Equilibrios del modelo}

Como la naturaleza juega con probabilidades de $1 / 4$ para $(B), 1 / 2$ para $(R)$ y $1 / 4$ para $(M)$, se emplean para obtener los valores esperados de los pagos del productor e intermediario en la parte final del juego; así, el

9 Los superíndices C indican "con contrato”. Los superíndices B, R, M significan "con clima bueno", "con clima regular" y "con clima malo", respectivamente. 
representado en la Figura 6 se puede reformular como el de la Figura 7. En ésta se observa que el intermediario elige $A$ en la parte superior del juego, mientras que en la inferior permanece indiferente entre optar por $A$ o $D$; podría proponerse que, en su turno, el productor considerara una distribución de probabilidad $(p, 1-p)$ para decidir entre No y $S i$, pero esto no es necesario porque $137 / 16200>1 / 128$, y $1 / 128=1 / 128$; entonces el productor siempre escogerá la estrategia $S i$; por lo tanto, hay dos EPS: $\{S i, A\},\{S i, D\}$. En este caso, cuando hay contratos anticipados y el intermediario carece de poder de mercado en el eslabón siguiente, tener poder de negociación se torna una estrategia de equilibrio para los productores; tales resultados no cambian al incrementarse las probabilidades de los estados $(B)$ y $(M)$ : se mantienen cuando los tres estados de la naturaleza tienen la misma probabilidad (1/3) - resultados no reportados - ; incluso se sostienen los mismos equilibrios al asignar la mayor probabilidad de ocurrencia $(1 / 2)$ a $(M)$ y mantener probabilidades iguales $(1 / 4)$ para los estados $(B)$ y $(R)$, con resultados tampoco reportados.

Si se contrasta este resultado con el del juego sin contratos y donde el intermediario tampoco cuenta con poder de mercado en el eslabón siguiente (ilustrado en la Figura 3), puede observarse que la existencia del contrato no modifica las combinaciones estratégicas de equilibrio, pero sí permite al productor obtener un pago mayor al que conseguirían sin el acuerdo. ${ }^{10}$ En cambio, los pagos esperados del intermediario son iguales a los de la situación sin contrato, ${ }^{11}$ en tanto éste contrarresta, en parte, la ventaja del poder de negociación del intermediario, quien desea seguir una estrategia de procuración de productos óptima en el tiempo que le permita cumplir con sus compromisos de ventas $y$, además, reducir problemas de selección adversa y riesgo moral relacionados con la calidad de lo comercializado. Esta situación beneficia los pagos de los productores; se trata de un resultado similar al obtenido por la literatura con enfoque de contratos y coordinación vertical (Sexton, 2013).

10 Los pagos del juego con contrato representan la diferencia respecto a los de la situación sin él, como se explica al final del apartado 2.2.2; como los pagos de equilibrio de la Figura 7 son positivos, entonces representan una diferencia positiva respecto a la situación sin contrato.

11 En concordancia con el comentario de la nota de pie de página anterior, los pagos de equilibrio iguales a cero en el juego de la Figura 7 representan, en cada caso, un pago esperado igual al de la situación sin contrato. 
Sólo cuando se asigna una probabilidad mayor $(1 / 2)$ al estado $(B)$ -y de 1/4 a los demás-, el equilibrio cambia a $\{$ No, $A\}$ (resultados no reportados), lo que puede significar que, ante una perspectiva muy favorable de condiciones climáticas, podría esperarse una mayor disponibilidad del producto a menor precio, por lo que no sería necesario para el intermediario ejercer poder de mercado en el proceso de compra del producto. En esta situación de formación de contratos, también se benefician los pagos esperados del productor.

Figura 6

JUEGO CON CONTRATO ANTICIPADO DONDE EL INTERMEDIARIO NO TIENE PODER DE MERCADO EN EL ESLABÓN SIGUIENTE

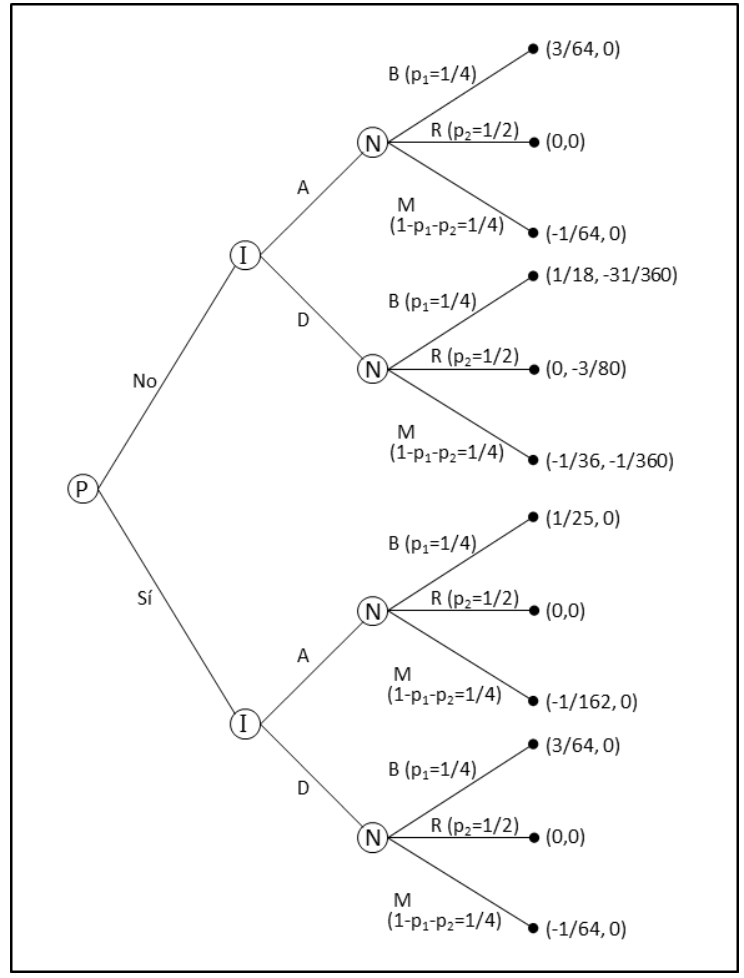

Fuente: Elaboración propia con base en las ecuaciones de los apartados 2.1.2.1 y 2.1.2.2. 
Figura 7

JUEGO DE LA FIGURA 6 DONDE SE HAN OBTENIDO LOS VALORES ESPERADOS DE LOS JUGADORES

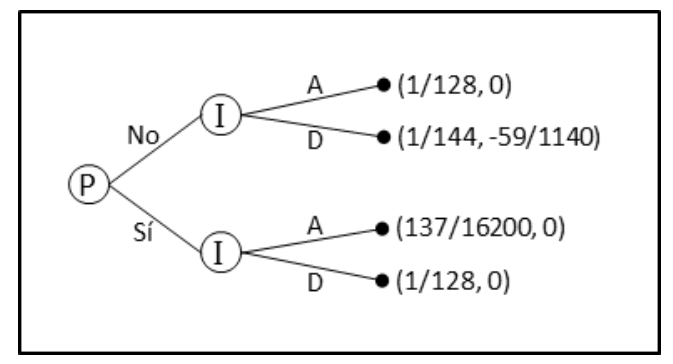

Fuente: Elaboración propia con base en las ecuaciones de los apartados 2.1.2.1 y 2.1.2.2.

\subsubsection{El intermediario sí tiene poder de mercado en el eslabón siguiente}

En este segundo caso de modelo de agricultura por contrato, el intermediario sí ejerce poder de mercado en el eslabón siguiente cuando entra al juego, es decir, maximiza sus beneficios de acuerdo con las ecuaciones 9 y 11, según corresponda; esto se presenta en la Figura 8.

\subsection{Equilibrios del modelo}

Como la naturaleza participa con probabilidades de $1 / 4$ para $(B), 1 / 2$ para $(R)$ y $1 / 4$ para $(M)$, se emplean para obtener los pagos esperados del productor e intermediario en la parte final del juego; de esta manera, la Figura 8 se puede reformular como el juego de la Figura 9, donde el intermediario elige $D$ en la parte superior del juego, mientras que en la inferior escoge $A$. Al considerar el turno del productor, a éste le resulta conveniente elegir $N o$, puesto que 9/512>7/1800; por lo tanto, hay un EPS: $\{N o, D\}$. Cabe señalar que estos resultados no se modifican al incrementarse las probabilidades de $(B)$ y $(M)$, ya que el equilibrio se sostiene para probabilidades iguales $(1 / 3)$ en todos los estados y aun cuando éstos tengan mayor probabilidad que los otros casos (resultados no reportados). 
Figura 8

JUEGO CON CONTRATO ANTICIPADO DONDE EL INTERMEDIARIO SÍ TIENE PODER DE MERCADO EN EL ESLABÓN SIGUIENTE

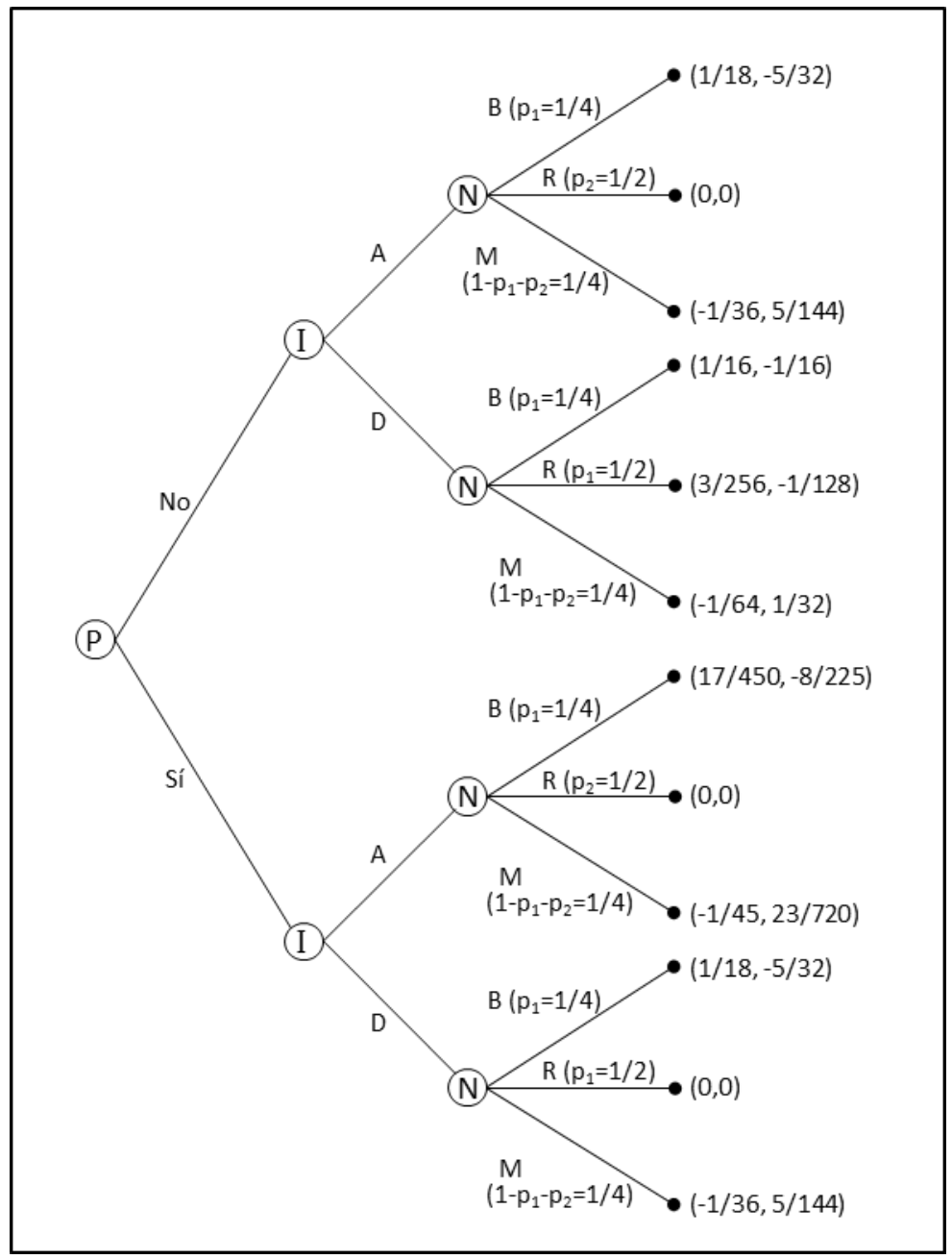

Fuente: Elaboración propia con base en las ecuaciones de los apartados 2.1.2.1 y 2.1.2.2. 
Figura 9

JUEGO DE LA FIGURA 8 DONDE SE HAN OBTENIDO LOS VALORES ESPERADOS DE LOS JUGADORES

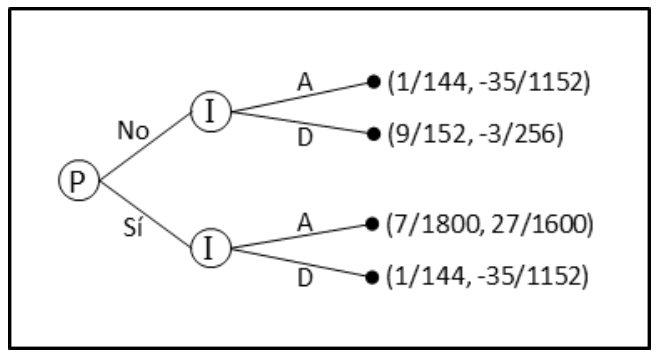

Fuente: Elaboración propia con base en las ecuaciones de los apartados 2.1.2.1 y 2.1.2.2.

Si se contrasta este resultado con el del juego sin contratos y donde el intermediario también cuenta con poder de mercado en el eslabón siguiente (ilustrado por la Figura 5), se observa que la existencia de este acuerdo sí modifica el sentido del equilibrio, ya que en el otro caso se obtenía $\{S i ́, D\}$, mientras que el contrato condujo a $\{N o, D\}$. Tal resultado destaca porque en este último equilibrio el intermediario, a pesar de que puede ejercer poder de mercado en ambas partes de la cadena de comercialización, obtiene pagos esperados inferiores a los de la situación sin contrato, mientras que para el productor son superiores. ${ }^{12}$ Al igual que en el modelo de la sección anterior (2.2.2.1), el contrato contrarresta parcialmente la ventaja del poder de negociación del intermediario y compensa al productor, con el propósito de que el primero asegure un proveedor confiable de mercancías que le permita cumplir con sus compromisos de ventas; así reduce problemas de selección adversa y riesgo moral relacionados con la calidad de los productos. Para lograr este tipo de acuerdos, el intermediario sacrifica una parte de sus ganancias. ${ }^{13}$

12 Debido a lo explicado en las dos notas de pie de página anteriores.

13 En el equilibrio $\{$ No, D $\}$ el intermediario obtiene un pago esperado de -3/256 (véase Figura 9), que tiene signo negativo porque resulta de comparar los beneficios con y sin contrato, aunque en realidad el intermediario conserva ganancias positivas iguales a 3/80. 


\section{CONCLUSIONES}

La conclusión más general del presente trabajo indica que, cuando un intermediario agrícola cuenta con poder de mercado en la fijación de su precio de venta, no sólo genera efectos en los eslabones siguientes de la cadena del producto, sino en el anterior: impactan en el productor debido a que, al ejercer el poder de mercado en la venta, adquiere menos cantidad de producto a menor precio; este resultado se mantiene aun cuando el productor tenga poder negociador en la venta, y a pesar de que el intermediario se comporte como precio-aceptante al comprar. Cuando además hay poder de mercado en la fijación del precio de compra, este efecto se refuerza, con el subsecuente incremento de los beneficios del intermediario y en perjuicio del productor. Si bien estos resultados podrían considerarse esperados, los modelos aquí presentados permiten identificar diversos detalles que, de otra forma, sería difícil observar.

En concreto, el conjunto de resultados de los modelos de teoría de juegos permite reconocer las relaciones entre el poder de mercado y las decisiones estratégicas de los productores e intermediarios agrícolas; se destacan, por tanto, los siguientes:

1) Cuando no hay contratos anticipados y el intermediario carece de poder de mercado en el eslabón siguiente, un resultado de equilibrio es que el productor busque tener poder de negociación, lo que implica actuar dentro de una organización o asociación de productores.

2) Cuando no hay contratos anticipados y el intermediario sí posee poder de mercado en el eslabón siguiente, un resultado de equilibrio es que el productor decida tener poder de negociación y el intermediario, poder de mercado sobre el precio al que compra. En este equilibrio ambas estrategias se contrarrestan parcialmente, pero cualquier desviación en la estrategia de equilibrio del productor conducirá a una situación en la que el intermediario ejerza poder de mercado en ambos eslabones. Si las condiciones socioculturales, geográficas o económicas impiden que un productor pertenezca a una organización o asociación destinada a comercializar mejor los productos, entonces el resultado será el peor posible para él.

3) Cuando se establecen contratos anticipados y el intermediario carece de poder de mercado en el eslabón siguiente, el contrato contrarresta parcialmente la ventaja del poder de negociación del interme- 
diario en provecho del productor. En este caso los beneficios del intermediario se mantienen como cuando no hay contrato.

4) Cuando se establecen contratos anticipados y el intermediario sí cuenta con poder de mercado en el eslabón siguiente, se logra un equilibrio en donde el productor carece de poder de negociación y, en cambio, el intermediario sí presenta poder de mercado en ambas partes de la cadena de comercialización. Sin embargo, el contrato provoca que éste se prive de una parte de sus ganancias (lo que no sucede en ausencia de tal acuerdo) para incentivar la participación del productor, en favor de los pagos de este último. Tal resultado concuerda con los obtenidos mediante un enfoque de contratos y coordinación vertical, donde se argumenta que los intermediarios sacrifican poder de mercado con el propósito de conseguir proveedores confiables que permitan cumplir con los compromisos de ventas, lo que reduce problemas de selección adversa y riesgo moral vinculados con la calidad de los productos.

El ejercicio de modelación aquí mostrado contribuye a valorar e interpretar la información y la evidencia empírica relacionadas con los mercados agrícolas, así como reflexionar sobre los diversos casos en donde los problemas de competencia económica pueden existir en perjuicio de los participantes de los mercados, aunque no sean evidentes o no estén tipificados por la legislación correspondiente. Al respecto, en la presente investigación los resultados ponen en relieve que la presencia de poder de mercado en cualquier parte de la cadena de intermediación en algún momento termina por alcanzar a los productores, quienes sustentan el sistema agroalimentario del país; de ahí la trascendencia de que las autoridades que regulan y supervisan al sector agrícola se enfoquen, con mayor profundidad, al estudio y seguimiento del fenómeno de la intermediación.

\section{Bibliografía}

Belleflamme, Paul y Peitz, Martin (2010), Industrial Organization: Markets and Strategies, Cambridge University Press, New York.

Bonanno, Alessandro; Russo, Carlo y Menapace, Luisa (2018), "Market Power and Bargaining in Agrifood Markets: A Review of Emerging Topics and Tools", en Agribusiness, 34, pp.6-23. 
Calleja-Pinedo, Margarita (2007), Intermediarios y comercializadores. Canales de distribución de frutas y hortalizas mexicanas en Estados Unidos, Universidad de Guadalajara/UCLA Program on Mexico/PROFMEX/Juan Pablos, Guadalajara.

Cofece (Comisión Federal de Competencia Económica) (2016), Reporte sobre las condiciones de competencia en el sector agroalimentario, Comisión Federal de Competencia Económica, México.

Condorelli, Daniele y Galeotti, Andrea (2016), "Strategic Models of Intermediation Networks", en Bramoulle, Y.; Galeotti, A. y Rogers, B. (eds.), The Oxford Handbook on the Economics of Networks, Oxford University Press, New York.

De la Rosa, Tomás (2016), "Té de manzanilla en México, ganancia de «coyotes»”, en EconomíaHoy.mx, <http:/www.economiahoy.mx/economiahoy/ opinion/noticias/7393300/03/16/Te-de-manzanilla-en-Mexico-gananciade-coyotes.html $>$ [02 de septiembre de 2017].

Eaton, Charles y Shepherd, Andrew W. (2002), Agricultura por contrato. Alianzas para el crecimiento, FAO, Roma, http://www.fao.org/3/y0937s/ y0937s01d.htm\#P29_13576 [10 de septiembre de 2017].

Manea, Mihai (2016), "Intermediation and Resale in Networks", Working Paper, Department of Economics/MIT, Massachusetts.

OECD (Organisation for Economic Co-operation and Development) (2016), OECD.Stat, <http://stats.oecd.org/Index.aspx?DatasetCode=MEI_PRICES\#>.

Pindyck, Robert S. y Rubinfeld, Daniel L. (2009), Microeconomics. Pearson Publishing, Malaysia.

Sexton, Richard J. (2013), "Market Power, Misconceptions, and Modern Agricultural Markets", en American Journal of Agricultural Economics, 95 (2), pp.209-219.

SIAP (Servicio de Información Agroalimentaria y Pesquera) (2015), "Márgenes de comercialización", en Gob.mx, Gobierno de México/ Secretaría de Agricultura, Ganadería, Desarrollo Rural, Pesca y Alimentación (Sagarpa), México, <http://www.gob.mx/siap/archivo/ documentos?order=DESC\&page=4 $>$ [20 de septiembre de 2017].

Spulber, Daniel F. (1996), "Market Microstructure and Intermediation", en Journal of Economic Perspectives, 10 (3), pp.135-152.

Spulber, Daniel F. (1999), Market Microstructure, Cambridge University Press, New York.

Tirole, Jean (1988), The Theory of Industrial Organization, MIT Press, Massachusetts. 


\section{APÉNDICE. EJEMPLO del CÁlCULO de LOS PAGOS DE LOS MODELOS DE TEORÍA DE JUEGOS}

En el presente apéndice se presentan dos ejemplos del cálculo de los pagos de los modelos del apartado 2 del documento:

1) Juego sin contratos anticipados en donde el intermediario no tiene poder de mercado en el eslabón siguiente:

Se presenta el cálculo de los pagos de la rama del juego $\{N o, D\}$ bajo el estado de la naturaleza $(B)$, tal como aparecen en las figuras 2 y 3 .

Dadas las características de esta rama del juego, la función de beneficios del intermediario es del tipo de la ecuación 5:

$$
\pi^{I}=p q-w(x) x-f
$$

Al usar la función de producción $q=f(x)=x$, se puede reformular la función de beneficios como una del tipo de la ecuación 10:

$$
\pi^{I}=p x-w(x) x-f
$$

Para el estado de la naturaleza $(B)$, la función de oferta del factor es $w(x)=x$. Al reemplazar se llega a la condición de primer orden:

$$
\frac{d \pi}{d x}=p-2 x=0
$$

Si se cambia $p$ por la función inversa de demanda $p(x)=1-x$, una vez resuelta se alcanzan los siguientes resultados:

$$
x^{*}=1 / 3, q^{*}=1 / 3, w^{*}=1 / 3, p^{*}=2 / 3
$$

Como $f=1 / 40$, se calcula el valor de la función de beneficios para el intermediario: 


$$
\pi^{I}=p^{*} q^{*}-w^{*} x^{*}-f
$$

$\mathrm{Al}$ sustituir los valores de equilibrio de las variables, se obtiene:

$$
\pi^{I}=(2 / 3)(1 / 3)-(1 / 3)(1 / 3)-1 / 40=\mathbf{3 1} / \mathbf{3 6 0}
$$

Con los mismos valores de equilibrio se calcula el valor de la función de beneficios para el productor, la cual sigue la forma de la ecuación 16:

$$
\pi^{P}=\hat{p} \hat{q}-\bar{c} \hat{q}
$$

Como $w^{*}$ y $x^{*}$ corresponden respectivamente a $p^{\wedge}$ y $q \hat{,}$, y $c=1 / 2$, entonces:

$$
\pi^{P}=(1 / 3)(1 / 3)-(1 / 2)(1 / 3)=-\mathbf{1} / \mathbf{1 8}
$$

2) Juego sin contratos anticipados en donde el intermediario sí tiene poder de mercado en el eslabón siguiente:

Se presenta el cálculo de los pagos de la rama del juego $\{S i, A\}$ bajo el estado de la naturaleza $(B)$, tal como aparecen en las figuras 4 y 5 .

Dadas las características de esta rama del juego, la función de beneficios del intermediario es del tipo de la ecuación 4:

$$
\pi^{I}=p(q) q-w x-f
$$

Al usar la función de producción $q=f(x)=x$, se reformula la función de beneficios como una del tipo de la ecuación 9:

$$
\pi^{I}=p(x) x-w x-f
$$


En esta rama del juego el productor sí cuenta con poder negociador en la determinación de los precios a los que vende al intermediario; implica que, por ejemplo, el productor pertenece a una organización de productores, de manera que puede beneficiarse de una negociación conjunta. En una rama del juego con las mismas características de la analizada, pero donde el productor no hubiera tenido poder negociador, se habría obtenido un $w^{*}=1 / 3$, que representaría $\hat{p}=1 / 3$ para el productor. Aquí se asume que, debido a su poder negociador, el productor obtendría un $\bar{p}$ ligeramente superior a $\hat{p}$, en este caso: $\bar{p}=2 / 5>\hat{p}$. El valor de $\bar{p}$ corresponde a uno dado $\bar{w}$ que el intermediario toma cuando es precio-aceptante.

La función de beneficios del intermediario se transforma en:

$$
\pi^{I}=p(x) x-2 / 5 x-f
$$

Si se cambia $p(x)$ por la función inversa de demanda $p(x)=1-x$, se llega a la condición de primer orden:

$$
\frac{d \pi}{d x}=1-2 x-2 / 5=0
$$

Se consiguen los siguientes resultados:

$$
x^{*}=3 / 10, q^{*}=3 / 10, p^{*}=7 / 10
$$

Como $f=1 / 40$, se calcula el valor de la función de beneficios para el intermediario:

$$
\pi^{I}=p^{*} q^{*}-\bar{w} x^{*}-f
$$

Al sustituir los valores de equilibrio de las variables, se obtiene:

$$
\pi^{I}=(7 / 10)(3 / 10)-(4 / 10)(3 / 10)-1 / 40=\mathbf{1 3} / \mathbf{2 0 0}
$$


Con los mismos valores de equilibrio se determina el valor de la función de beneficios para el productor, la cual sigue la forma de la ecuación 17:

$$
\pi^{P}=\bar{p} \hat{q}-\bar{c} \hat{q}
$$

Como $x^{*}$ corresponde a $\hat{q}$, y como $\bar{p}=2 / 5, \bar{c}=1 / 2$, entonces:

$$
\pi^{P}=(2 / 5)(3 / 10)-(1 / 2)(3 / 10)=-\mathbf{3} / \mathbf{1 0 0}
$$

